\title{
TERAPIA FONOLÓGICA: A GENERALIZAÇÃO DENTRO DE UMA CLASSE DE SONS E PARA OUTRAS CLASSES DE SONS
}

\author{
Phonological therapy: the generalization inside a sound class \\ and for other sound classes
}

Marizete Ilha Ceron (1), Márcia Keske-Soares ${ }^{(2)}$

\begin{abstract}
RESUMO
Objetivo: comparar a generalização dentro de uma classe e para outras classes de sons no tratamento de sujeitos com diferentes gravidades do desvio fonológico submetidos aos Modelos de Ciclos Modificado, ABAB-Retirada e Provas Múltiplas e Oposições Máximas Modificado. Métodos: 21 crianças com desvio fonológico, 11 do sexo masculino e 10 do feminino, com média de idade de 5:7 anos tiveram seus dados de fala foram analisados por meio da avaliação fonológica. Após a realização da análise contrastiva, foi calculado o Percentual de Consoantes Correta (Shriberg e Kwiatkowski, 1982) e os sujeitos foram classificados em diferentes gravidades do desvio fonológico: grave, moderadograve, leve-moderado, leve. Seis sujeitos foram submetidos ao Modelo de Ciclos Modificado, oito ao ABAB-Retirada e Provas Múltiplas, e sete ao de Oposições Máximas Modificado. Fez-se uma comparação da generalização dentro de uma classe e para outras classes de sons entre os três modelos em cada grau de gravidade do desvio fonológico. Resultados: a generalização dentro de uma classe de sons ocorreu em todos os modelos pesquisados. O Modelo ABAB-Retirada e Provas Múltiplas foi efetivo para todos os graus de gravidade e o de Oposições Máximas Modificado foi mais efetivo nos graus grave e moderado-grave. Em relação à generalização para outras classes de sons os sujeitos submetidos ao Modelo ABAB-Retirada e Provas Múltiplas foram os que apresentaram um maior número de produções corretas nas diferentes gravidades do desvio fonológico. Conclusão: todos os sujeitos apresentaram evoluções quanto às generalizações pesquisadas nos três modelos.
\end{abstract}

DESCRITORES: Fonoterapia; Generalização; Fala; Distúrbios da Fala

\section{INTRODUÇÃO}

A terapia com base fonológica centra-se na reorganização do sistema fonológico da criança, tendo como principal objetivo a generalização ${ }^{1-3}$.

A generalização é a ampliação da produção e uso correto dos fones-alvo treinados em outros contextos ou ambientes não-treinados. A generalização pode ser analisada pelo enfoque estrutural ou funcional. O primeiro refere-se à identificação das cir-

(1) Fonoaudióloga; Bolsista da Coordenação de Aperfeiçoamento de Pessoal de Nível Superior; Especialização em Fonoaudiologia pela Universidade Federal de Santa Maria; Mestranda em Distúrbios da Comunicação Humana pela Universidade Federal de Santa Maria.

(2) Fonoaudióloga; Professora do Curso de Fonoaudiologia e do Programa de Pós-Graduação em Distúrbios da Comunicação Humana da Universidade Federal de Santa Maria; Doutora em Lingüística Aplicada pela Pontifícia Universidade Católica do Rio Grande do Sul. cunstâncias sob as quais ela ocorre, enquanto que o segundo é o modo pelo qual cada criança reorganiza seu sistema fonológico. Alguns dos componentes estruturais da generalização são: a itens não utilizados no tratamento (outras palavras); para outra posição na palavra; dentro de uma classe de sons; para outras classes de sons ${ }^{4}$.

Nesta pesquisa será enfocado apenas à generalização dentro de uma classe de sons e para outras classes de sons. Na primeira a criança aprende um som e estende este aprendizado a outros sons da mesma classe estimulada; na segunda, as características dos sons aprendidos são refletidas para sons de outras classes não trabalhadas em terapia. As generalizações são importantes para diminuir o tempo de terapia fonológica. Pois, esse tipo de generalização indica que os sons se relacionam entre si e podem ser adquiridos sem que haja uma intervenção direta em todos eles ${ }^{1}$. 
A possibilidade de estimular a generalização foi a principal contribuição dos modelos fonológi$\cos ^{2,3}$, uma vez que leva a uma terapia mais eficiente, eliminando a difícil tarefa de ensinar todos os sons incorretos em todas as palavras, contextos ou ambientes ${ }^{1}$.

Os modelos fonológicos têm em comum a noção de reorganização do sistema fonológico com desvios, entre os modelos existentes destaca-se: 0 Modelo de Ciclos Modificado 5 , o Modelo ABABRetirada e Provas Múltiplas ${ }^{6}$ e o Modelo Oposições Máximas Modificado ${ }^{7}$.

O Modelo de Ciclos Modificado tem por objetivo facilitar a emergência de novos padrões de sons através do tratamento em ciclos, baseando em processos fonológicos ${ }^{8}$. A seleção de padrões alvo para o ciclo de tratamento permite reforçar 0 desenvolvimento do sistema fonológico da criança e, assim, agilizar seus ganhos de inteligibilidade ${ }^{9}$.

O Modelo ABAB-Retirada e Provas Múltiplas é uma proposta baseada na hierarquia implicacional de traços distintivos para a escolha dos sons-alvo de tratamento. O princípio terapêutico indica que o tratamento de sons mais difíceis facilita a mudanças no sistema fonológico da criança sem o tratamento direto de sons mais fáceis ${ }^{10}$.

O Modelo de Oposições Máximas Modificado ${ }^{7}$ tem como procedimento o contraste de oposições máximas, isto é, palavras que diferem em apenas um fonema (som), os quais se diferenciam em dois ou mais traços distintivos. O propósito deste modelo é fazer com que a criança reorganize seu sistema fonológico através da percepção auditiva, imitação da produção e produção espontânea das palavrasalvo, sendo realizada em duas etapas: imitação e produção espontânea.

$\mathrm{Na}$ literatura encontram-se descrições ${ }^{5-7,11-14}$ de várias abordagens de intervenção fonológica para o desvio fonológico, mas estudos ${ }^{1,8,15-17}$ que comparam os modelos de terapia são poucos.

Algumas pesquisas ${ }^{1,3,8,10,18-21} \mathrm{com}$ modelos fonológicos que relataram a presença da generalização dentro de uma classe de sons e para outras classes de sons no tratamento de crianças com desvio fonológico (DF).

Foram poucos os estudos ${ }^{1,8,20}$ encontrados que comparam a generalização dentro de uma classe de sons e para outras classes de sons obtidas em diferentes modelos de tratamento e gravidades, sendo necessários novos trabalhos, pois essas pesquisas podem auxiliar o clínico na escolha de modelos de terapia mais eficazes para cada caso.

Portanto, o objetivo deste estudo é comparar a generalização dentro de uma classe e para outras classes de sons no tratamento de sujeitos com diferentes gravidades do desvio fonológico sub- metidos aos Modelos de Ciclos Modificado, ABABRetirada e Provas Múltiplas e Oposições Máximas Modificado.

\section{MÉTODOS}

Os dados utilizados fazem parte de três dissertações de mestrado do Programa de Pós-graduação em Distúrbios da Comunicação Humana da Universidade Federal de Santa Maria. A primeira ${ }^{18}$ tinha como objetivo verificar a generalização em sujeitos com diferentes gravidades do DF tratados pelo Modelo de Ciclos Modificado; a segunda 19 tinha o objetivo de analisar a generalização obtida pelo Modelo ABAB-Retirada e Provas Múltiplas em crianças com diferentes gravidades do DF; e a terceira ${ }^{21}$ tinha como um de seus objetivos analisar as mudanças fonológicas ocorridas em sujeitos com diferentes gravidades do DF tratados pelo Modelo Oposições Máximas Modificado. Para que os sujeitos participassem da pesquisa, os pais ou responsáveis assinaram o Termo de Consentimento Livre e Esclarecido autorizando as pesquisadoras à realização de seus estudos.

O grupo pesquisado foi constituído por 21 sujeitos, sendo dez do sexo feminino e onze do masculino, cuja média de idade no início do tratamento era de 5:7 anos. Os sujeitos foram tratados com três diferentes modelos de terapia fonológica: seis (C3 ao C8) submetidos ao Modelo de Ciclos Modificado 5; oito (A1 ao A8) pelo ABAB-Retirada e Provas Múltiplas ${ }^{6}$; e sete (O1 ao O8) ao de Oposições Máximas Modificado ${ }^{7}$. Para cada modelo terapêutico havia dois sujeitos para cada grau de gravidade. No grupo tratado pelo Modelo de Oposições Máximas Modificado, um dos sujeitos com desvio moderado-grave (O4) foi excluído ${ }^{21}$, pelo fato de seu sistema fonológico não permitir a escolha de sons-alvo compatíveis com sua proposta. No Modelo de Ciclos Modificado, a pesquisadora ${ }^{18}$ não encontrou sujeitos com desvio grave para participar da pesquisa.

No Modelo de Ciclos Modificado, a terapia teve duração de no máximo dois ciclos de tratamento de três semanas, sendo realizadas duas sessões semanais de 50 minutos. No Modelo ABAB-Retirada e Provas Múltiplas, a intervenção terapêutica teve duração de aproximadamente cinco semanas (nove sessões), sendo realizadas duas sessões por semana de 45 minutos. No Modelo de Oposições Máximas Modificado, foram realizadas duas sessões semanais, com duração de 45 minutos, totalizando 20 sessões de terapia.

Para constituir o grupo pesquisado, conforme os critérios de inclusão utilizados pelas pesquisadoras, os sujeitos deveriam apresentar: diagnóstico prévio de DF na triagem realizada no serviço de atendimento 
fonoaudiológico da instituição; ausência de alterações significativas nas avaliações realizadas, exceto na fonológica; diferentes gravidades do DF, segundo a classificação do Percentual de Consoantes Corretas ${ }^{22}$ (PCC); e não ter sido submetido à terapia fonoaudiológica anteriormente a este estudo.

O diagnóstico de DF das pesquisas utilizadas foi realizado a partir da submissão das crianças às seguintes avaliações: informal de linguagem; do sistema estomatognático; psicomotora; de discriminação auditiva; além da avaliação fonológica da criança ${ }^{23}$ (AFC). Foram realizados exames complementares (avaliação otorrinolaringológica, audiológica e neurológica) em todos os sujeitos para descartar outros comprometimentos.

A partir da avaliação fonológica, na análise contrastiva, foi possível determinar o sistema fonológico de cada sujeito. Para fins de análise do sistema fonológico ${ }^{24}$, foi considerado que o fonema estava estabelecido quando ocorreu de $80 \%$ a $100 \%$ das vezes, parcialmente adquirido quando ocorreu de $40 \%$ - $79 \%$, e não adquirido quando ocorre de $0 \%-39 \%$ das possibilidades.

Para constituir a amostra da pesquisa, os sujeitos foram classificados em diferentes gravidades do DF, calculados a partir do PCC. Os sujeitos foram classificados em grupos com: desvio grave, com PCC menor que 50\%; desvio moderado-grave, com PCC de 51 a $65 \%$; desvio leve-moderado, com PCC entre 66 e $85 \%$; e desvio leve, com PCC de 86 a $100 \%$.

Os sujeitos A1, A2, $\mathrm{O} 1$ e $\mathrm{O} 2$ foram classificados como com desvio grave; $\mathrm{C} 3, \mathrm{C} 4, \mathrm{~A} 3, \mathrm{~A} 4$ e $\mathrm{O} 3 \mathrm{com}$ desvio moderado-grave; $\mathrm{C} 5, \mathrm{C} 6, \mathrm{~A} 5, \mathrm{~A} 6, \mathrm{O} 5$ e $\mathrm{O} 6$ os com desvio leve-moderado; e C7, C8, A7, A8, $\mathrm{O} 7$ e $\mathrm{O} 8$ os com desvio leve, sendo consideradas as letras iniciais a referência ao modelo terapêutico, ou seja, "O" os que foram tratados pelo Modelo de Oposições Máximas Modificado, "A" os que foram tratados pelo ABAB-Retirada e Provas Múltiplas, e "C" os que foram tratados pelo Modelo de Ciclos Modificado.

Esta pesquisa está vinculada ao projeto de pesquisa aprovado pelo Comitê de Ética em Pesquisa (CEP) de uma instituição de ensino superior sob no 046/02.

Esta pesquisa é descritiva, sendo realizada uma análise qualitativa da generalização dentro de uma classe de sons e para outras classes de sons. As mesmas foram consideradas quando outros sons de uma mesma classe estimulada ou de outras classes não trabalhadas foram produzidas corretamente.

A fim de comparar esta generalização entre os modelos terapêuticos em diferentes gravidades do DF analisou-se qualitativamente o som-alvo, os outros sons da mesma classe e de outras classes, verificou-se o aumento do percentual de acertos na avaliação final de cada sujeito.

\section{RESULTADOS}

Os alofones [t $\left.\int\right]$, [d3] serão referenciados entre colchetes, para diferenciá-los dos demais fonemas, uma vez que não são fones contrastivos no Português.

Na Tabela 1, são apresentados os resultados da generalização dentro de uma classe de sons encontrada nos sujeitos do grupo com desvio grave e com desvio moderado-grave submetidos aos Modelos de Ciclos Modificado, ABAB-Retirada e Provas Múltiplas e Oposições Máximas Modificado.

Dos sujeitos com desvio grave, o A1 generalizou apenas para a classe das africadas. Esse sujeito, no entanto, não apresentou generalização para a classe das líquidas. O A2 aumentou o número de produções corretas para a classe das líquidas e fricativas. O O2 apresentou este tipo de generalização para a líquida /R/ e para a fricativa $/ \mathrm{V} /$, não generalizou para as fricativas $/ 3 /$ e $/ \mathrm{J} /$, nem para a líquida / $\mathrm{K} /$. O 01 também generalizou, principalmente para as líquidas e fricativas.

No grupo com desvio moderado-grave, constatou-se que apenas o sujeito C3 e o A4 não apresentaram generalização dentro de uma mesma classe de sons, para alguns dos sons possíveis. Ao contrário do C4 e A3 que não apresentavam possibilidades de ocorrência em algumas classes de sons, como na classe das líquidas para o A3.

O C3 generalizou principalmente para a classe das plosivas. Esse sujeito, no entanto, não apresentou generalização para fricativa e líquida. O C4 teve possibilidade de generalizar apenas para a fricativa $/ \int /$, não apresentou possibilidade de produções corretas para a classe das líquidas. O A3 generalizou, principalmente, para a plosiva /k/. O A4 apresentou possibilidade de produções corretas para a classe das líquidas.

A Tabela 2 mostra os resultados da generalização dentro de uma classe de sons encontrada nos sujeitos com desvio leve-moderado e leve submetidos a diferentes abordagens terapêuticas. Observase que todos os sujeitos apresentaram aumento das produções corretas dos sons pertencentes à mesma classe dos sons-alvo, em todos os modelos de terapia pesquisados.

Dos sujeitos com desvio leve-moderado, os que mais generalizaram foram o sujeito $A 5$, seguido do O5 e C5, tratados, respectivamente, pelos Modelos ABAB-Retirada e Provas Múltiplas, Oposições Máximas Modificado e Ciclos Modificado. O A5 e o O5 apresentaram um maior aumento do percentual 
Tabela 1 - Generalização dentro de uma classe de sons no tratamento do grupo Grave e Moderado-Grave

\begin{tabular}{|c|c|c|c|c|c|}
\hline Grau & Sujeito & $\begin{array}{c}\text { Classe } \\
\text { estimulada } \\
\text { (som-alvo) }\end{array}$ & $\begin{array}{l}\text { Sons não } \\
\text { estimulados }\end{array}$ & Al & AF \\
\hline \multirow{4}{*}{ Grave } & $\mathrm{A} 1$ & 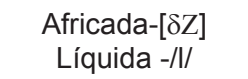 & $\begin{array}{c}{[\tau \Sigma]} \\
|\times|,|r|, / R /\end{array}$ & $\begin{array}{l}0,00 \\
0,00\end{array}$ & $\begin{array}{c}81,48 \\
0,00\end{array}$ \\
\hline & A2 & $\begin{array}{l}\text { Líquidas -/×/ } \\
\text { Líquida- /r/ } \\
\text { Fricativa -/Z/ }\end{array}$ & $\begin{array}{c}\text { /I/ } \\
/ \mathrm{r} / \\
/ \mathrm{R} / \\
/ / \\
/ \mathrm{I} / \\
/ \mathrm{R} / \\
/ \mathrm{f} / \\
/ \mathrm{v} / \\
/ \mathrm{s} /(\mathrm{O}) \\
/ \mathrm{s} /(\mathrm{C}) \\
/ \mathrm{S} /\end{array}$ & $\begin{array}{c}12,07 \\
0,00 \\
76,00 \\
12,07 \\
0,00 \\
76,00 \\
0,00 \\
38,47 \\
0,00 \\
48,28 \\
0,00\end{array}$ & $\begin{array}{l}59,70 \\
50,00 \\
93,55 \\
59,70 \\
68,75 \\
93,55 \\
97,22 \\
97,73 \\
98,67 \\
95,45 \\
80,00\end{array}$ \\
\hline & 01 & $\begin{array}{l}\text { Fricativa -/s/ } \\
\text { Líquida }-/ \times /\end{array}$ & $\begin{array}{l}|\mathrm{z}| \\
|\mathrm{Z}| \\
/ \mathrm{R} / \\
/ \mathrm{I} / \\
/ \mathrm{r} /\end{array}$ & $\begin{array}{c}0,00 \\
20,00 \\
28,57 \\
6,66 \\
0,00\end{array}$ & $\begin{array}{c}25,00 \\
16,66 \\
100,00 \\
70,00 \\
7,14\end{array}$ \\
\hline & $\mathrm{O2}$ & $\begin{array}{l}\text { Fricativa -/z/ } \\
\text { Líquida -/r/ }\end{array}$ & $\begin{array}{l}\mid \mathrm{s} / \\
/ \mathrm{v} / \\
/ \Sigma / \\
\mid \mathrm{Z} / \\
/ \mathrm{R} / \\
/ \mathrm{I} / \\
|\times|\end{array}$ & $\begin{array}{c}55,55 \\
20,00 \\
100,00 \\
0,00 \\
0,00 \\
0,00 \\
0,00\end{array}$ & $\begin{array}{c}85,71 \\
100,00 \\
0,00 \\
0,00 \\
100,00 \\
33,33 \\
0,00\end{array}$ \\
\hline \multirow{5}{*}{ Moderado-Grave } & C3 & $\begin{array}{l}\text { Plosiva -/g/ } \\
\text { Fricativa -/Z/ } \\
\text { Líquida -/R/ }\end{array}$ & $\begin{array}{l}/ \mathrm{b} / \\
/ \mathrm{d} / \\
/ \mathrm{f} / \\
/ \mathrm{v} / \\
/ \mathrm{s} / \\
/ \mathrm{z} / \\
/ \mathrm{I} / \\
/ \times 1 \\
/ \mathrm{r} /\end{array}$ & $\begin{array}{c}13,64 \\
25,92 \\
45,45 \\
0,00 \\
29,03 \\
14,28 \\
72,00 \\
0,00 \\
0,00\end{array}$ & $\begin{array}{c}85,72 \\
100,00 \\
0,00 \\
0,00 \\
31,25 \\
1428 \\
81,25 \\
0,00 \\
5,55\end{array}$ \\
\hline & $\mathrm{C} 4$ & $\begin{array}{l}\text { Fricativas-/s/ e /z/ } \\
\text { Líquidas - /l/, /×I, } \\
\text { /R/, /r/ }\end{array}$ & $\begin{array}{l}/ \Sigma / \\
\mathrm{s} / \mathrm{o}\end{array}$ & $\begin{array}{c}77,78 \\
-\end{array}$ & $\begin{array}{c}88,88 \\
-\end{array}$ \\
\hline & A3 & $\begin{array}{l}\text { Plosiva- /g/ } \\
\text { Líquida -/r/ }\end{array}$ & $\begin{array}{l}/ \mathrm{b} / \\
/ \mathrm{d} / \\
/ \mathrm{k} / \\
*\end{array}$ & $\begin{array}{c}52,63 \\
21,21 \\
7,69 \\
\text { * }\end{array}$ & $\begin{array}{c}54,67 \\
40,69 \\
100,00 \\
*\end{array}$ \\
\hline & A4 & $\begin{array}{l}\text { Líquida -/R/ } \\
\text { Líquida -/×I } \\
\text { Líquida -/r/ }\end{array}$ & $\begin{array}{c}/ / / \\
|\times| \\
\mid r /(O) \\
/ r /(C) \\
/ I / \\
\mid r /(O) \\
/ r /(C) \\
|\times| \\
/ I /\end{array}$ & $\begin{array}{c}18,92 \\
20,00 \\
4,26 \\
68,75 \\
18,92 \\
4,26 \\
68,75 \\
20,00 \\
18,92\end{array}$ & $\begin{array}{l}80,00 \\
80,00 \\
91,67 \\
46,51 \\
80,00 \\
91,67 \\
46,51 \\
80,00 \\
80,00\end{array}$ \\
\hline & 03 & Líquidas - /R/ e /I/ & $\begin{array}{l}|x| \\
|r|\end{array}$ & $\begin{array}{l}0,00 \\
0,00\end{array}$ & $\begin{array}{c}87,50 \\
100,00\end{array}$ \\
\hline
\end{tabular}

Legenda: Al: avaliação inicial. AF: avaliação final. O: onset . C: coda. s/o: sem possibilidade de ocorrência. *não houve possibilidade de ocorrência devido aos demais sons da classe não apresentarem alterações na Al 
Tabela 2 - Generalização dentro de uma classe de sons no tratamento do grupo Leve-Moderado e Leve

\begin{tabular}{|c|c|c|c|c|c|}
\hline Modelo & Sujeito & $\begin{array}{c}\text { Classe } \\
\text { estimulada } \\
\text { (som-alvo) }\end{array}$ & $\begin{array}{l}\text { Sons não } \\
\text { estimulados }\end{array}$ & $\mathrm{Al}$ & AF \\
\hline \multirow{6}{*}{ Leve - Moderado } & C5 & $\begin{array}{l}\text { Plosiva -/g/ } \\
\text { Líquida -/r/ }\end{array}$ & $\begin{array}{c}/ \mathrm{k} / \\
*\end{array}$ & $\underset{*}{16,00}$ & $\underset{*}{100,00}$ \\
\hline & C6 & $\begin{array}{l}\text { Plosiva -/g/ } \\
\text { Fricativa -/Z/ } \\
\text { Líquida -/r/ }\end{array}$ & $\begin{array}{c}/ \mathrm{b} / \\
|\mathrm{z}| \\
*\end{array}$ & $\begin{array}{c}78,57 \\
38,89 \\
*\end{array}$ & $\begin{array}{c}100,00 \\
100,00 \\
*\end{array}$ \\
\hline & A5 & Fricativa -/Z/ & $\begin{array}{c}/ \mathrm{v} / \\
/ \mathrm{s} /(\mathrm{O}) \\
/ \mathrm{s} /(\mathrm{C}) \\
|\mathrm{z}| \\
\mid \Sigma /\end{array}$ & $\begin{array}{c}75,00 \\
0 \\
0 \\
0 \\
0\end{array}$ & $\begin{array}{c}100,00 \\
39,39 \\
0,00 \\
26,67 \\
92,31\end{array}$ \\
\hline & A6 & Líquida -/R/ & $\begin{array}{l}/ \mathrm{r} /(\mathrm{O}) \\
/ \mathrm{r} /(\mathrm{C})\end{array}$ & $\begin{array}{l}0 \\
0\end{array}$ & $\begin{array}{c}0,00 \\
21,43\end{array}$ \\
\hline & 05 & Fricativa -/z/ & $/ \mathrm{s} /$ & 1,85 & 87,50 \\
\hline & 06 & $\begin{array}{l}\text { Plosiva -/g/ } \\
\text { Fricativa -/Z/ }\end{array}$ & $\begin{array}{l}/ \mathrm{b} / \\
/ \mathrm{d} / \\
/ \mathrm{v} / \\
\mid \mathrm{z} / \\
/ \Sigma /\end{array}$ & $\begin{array}{c}66,66 \\
55,00 \\
35,00 \\
40,00 \\
100,00\end{array}$ & $\begin{array}{c}83,33 \\
100,00 \\
100,00 \\
75,00 \\
75,00\end{array}$ \\
\hline \multirow{6}{*}{ Leve } & C7 & $\begin{array}{l}\text { Líquida }-/ r \text { l } \\
\text { OM } \\
\text { e CM }\end{array}$ & * & * & * \\
\hline & C8 & $\begin{array}{l}\text { Fricativa -/f/ } \\
\text { Líquida -/r/ }\end{array}$ & $\stackrel{*}{*}$ & $\stackrel{*}{*} 20$ & $\stackrel{*}{100}$ \\
\hline & A7 & $\begin{array}{l}\text { Fricativas-/Z/ } \\
\text { Líquida -/r/ }\end{array}$ & $\begin{array}{c}\mid \Sigma / \\
\mid s /(\mathrm{C}) \\
|z| \\
|\times|\end{array}$ & $\begin{array}{l}30,56 \\
64,58 \\
85,11 \\
46,15\end{array}$ & $\begin{array}{l}96,87 \\
93,33 \\
95,15 \\
80,36\end{array}$ \\
\hline & A8 & $\begin{array}{c}\text { Líquida -/rl } \\
\text { OM } \\
\text { e CM }\end{array}$ & * & * & * \\
\hline & 07 & $\begin{array}{l}\text { Líquida -/r/ } \\
\text { Nasal -/n/ }\end{array}$ & * & * & * \\
\hline & 08 & Africada $-[\tau \Sigma]$ & {$[\delta \mathrm{Z}]$} & 16,66 & 40,00 \\
\hline
\end{tabular}

Legenda: Al: avaliação inicial. AF: avaliação final. O: onset. C: coda. OM: onset medial. CM: coda medial. *não houve possibilidade de ocorrência devido aos demais sons da classe não apresentarem alterações.

para as fricativas, enquanto que o C5 apresentou para a classe das plosivas.

Do grupo com desvio leve, dos sujeitos que tinham possibilidades de generalizar, o que mais aumentou o percentual de acertos das produções foi o A7 para a classe das fricativas. Porém, os sujeitos C7, A8 e 07 não apresentaram possibilidades de generalizar devido aos demais sons da classe não apresentarem alterações.

$\mathrm{Na}$ Tabela 3, são apresentados os resultados da generalização para outras classes de sons nos sujeitos com desvio grave e moderado-grave submetidos a diferentes abordagens terapêuticas
(Ciclos Modificado, ABAB-Retirada e Provas Múltiplas e Oposições Máximas Modificado).

Nota-se que todos os sujeitos com desvio grave e moderado-grave apresentaram um aumento das produções corretas para a maioria dos sons pertencentes a outras classes de sons. Exceto o C3 que não teve possibilidade de generalizar, pois as únicas classes que apresentavam alterações foram tratadas (plosiva, fricativa e líquida). O sujeito que apresentou um maior número de produções corretas em outras classes de sons não tratadas foi - A2, tratado pelo Modelo ABAB-Retirada e Provas Múltiplas, no grupo grave e o A3 no grupo 
Tabela 3 - Generalização para outra classe de sons no tratamento do grupo Grave e Moderado-Grave

\begin{tabular}{|c|c|c|c|c|c|}
\hline \multirow{2}{*}{ Grau } & \multirow{2}{*}{ Sujeito } & \multirow{2}{*}{$\begin{array}{c}\text { Classes } \\
\text { estimuladas }\end{array}$} & \multirow{2}{*}{$\begin{array}{l}\text { Classes não } \\
\text { estimuladas }\end{array}$} & \multicolumn{2}{|c|}{$\%$ de acertos } \\
\hline & & & & Al & AF \\
\hline \multirow{4}{*}{ Grave } & A1 & $\begin{array}{l}\text { Africada [ }[\delta Z] \\
\text { Líquida /I/ }\end{array}$ & $\begin{array}{c}\text { Plosivas /p/ } \\
/ \mathrm{b} / \\
/ \mathrm{t} / \\
/ \mathrm{d} / \\
\text { Nasais /m/ } \\
/ \mathrm{n} / \\
\text { Fricativas /f/ } \\
/ \mathrm{v} / \\
/ \mathrm{s} /(\mathrm{O}) \\
/ \mathrm{s} /(\mathrm{C}) \\
/ \Sigma / \\
/ \mathrm{Z} / \\
\text { Africada }[\tau \Sigma][\delta \mathrm{Z}]\end{array}$ & $\begin{array}{c}30,77 \\
15,00 \\
0,00 \\
5,26 \\
23,08 \\
13,33 \\
20,00 \\
64,29 \\
70,59 \\
30,00 \\
0,00 \\
0,00 \\
0,00 \\
0,00\end{array}$ & $\begin{array}{c}100,00 \\
100,00 \\
86,00 \\
77,59 \\
91,67 \\
73,17 \\
100,00 \\
100,00 \\
96,23 \\
72,73 \\
46,15 \\
38,46 \\
81,48 \\
78,26\end{array}$ \\
\hline & A2 & $\begin{array}{l}\text { Líquidas } / \times / \text { e } \\
\qquad / \mathrm{r} / \\
\text { Fricativa } / \mathrm{Z} /\end{array}$ & $\begin{array}{c}\text { Plosivas /p/ } \\
/ \mathrm{b} / \\
/ \mathrm{t} / \\
/ \mathrm{d} / \\
/ \mathrm{k} / \\
/ \mathrm{g} / \\
\text { Nasais } / \mathrm{m} / \\
/ \mathrm{n} / \\
/ \mathrm{N} / \\
\text { Fricativas /f/ } \\
/ \mathrm{v} / \\
/ \mathrm{s} /(\mathrm{O}) \\
/ \mathrm{s} /(\mathrm{C}) \\
/ \Sigma / \\
/ \mathrm{Z} / \\
\text { Africada }[\tau \Sigma][\delta \mathrm{Z}]\end{array}$ & $\begin{array}{c}55,26 \\
25,93 \\
0,00 \\
9,09 \\
7,89 \\
0,00 \\
66,67 \\
58,62 \\
43,75 \\
0,00 \\
38,47 \\
0,00 \\
48,28 \\
0,00 \\
0,00 \\
6,67 \\
0,00\end{array}$ & $\begin{array}{c}97,10 \\
94,12 \\
100,00 \\
100,00 \\
99,05 \\
96,77 \\
98,15 \\
96,15 \\
100,00 \\
100,00 \\
100,00 \\
100,00 \\
97,87 \\
96,15 \\
100,00 \\
100,00 \\
97,10\end{array}$ \\
\hline & 01 & $\begin{array}{l}\text { Fricativa /s/ } \\
\text { Líquida / × }\end{array}$ & $\begin{array}{c}\text { Plosivas /b/ } \\
\text { /d/ } \\
/ \mathrm{g} / \\
\text { Nasal } / \mathrm{n} / \\
\text { Africada }[\delta \mathrm{Z}]\end{array}$ & $\begin{array}{c}10,00 \\
21,42 \\
0,00 \\
33,33 \\
0,00\end{array}$ & $\begin{array}{c}15,78 \\
53,33 \\
0,00 \\
80,00 \\
0,00\end{array}$ \\
\hline & $\mathrm{O} 2$ & $\begin{array}{l}\text { Fricativa /z/ } \\
\text { Líquida /r/ }\end{array}$ & $\begin{array}{c}\text { Plosivas /b/ } \\
/ \mathrm{d} / \\
/ \mathrm{g} / \\
\text { Africada }[\delta \mathrm{Z}] \\
\text { Nasal /// }\end{array}$ & $\begin{array}{c}12,50 \\
0,00 \\
0,00 \\
0,00 \\
33,33\end{array}$ & $\begin{array}{c}60,00 \\
87,50 \\
0,00 \\
100,00 \\
66,66\end{array}$ \\
\hline \multirow{5}{*}{ Moderado-Grave } & $\mathrm{C} 3$ & $\begin{array}{l}\text { Plosiva /g/ } \\
\text { Fricativa /Z/ } \\
\text { Líquida /r/ }\end{array}$ & $*$ & * & * \\
\hline & $\mathrm{C} 4$ & $\begin{array}{c}\text { Fricativa } / \mathrm{s} / \mathrm{e} / \mathrm{z} / \\
\text { Líquidas } / 1 /, / \mathrm{R} / \text {, } \\
\text { /r/ e } / \times /\end{array}$ & $\begin{array}{c}\text { Africada }[\delta Z] \\
{[\tau \Sigma]} \\
\text { Nasal } / \mathrm{n} /\end{array}$ & $\begin{array}{l}75,00 \\
11,11 \\
80,00\end{array}$ & $\begin{array}{c}25,00 \\
0,00 \\
100,00\end{array}$ \\
\hline & A3 & $\begin{array}{l}\text { Plosiva /g/ } \\
\text { Líquida /r/ }\end{array}$ & 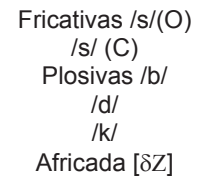 & $\begin{array}{c}41,94 \\
50,00 \\
52,63 \\
21,21 \\
7,69 \\
75,00\end{array}$ & $\begin{array}{c}74,71 \\
66,67 \\
54,67 \\
40,69 \\
100,00 \\
28,57\end{array}$ \\
\hline & A4 & $\begin{array}{c}\text { Líquidas } / R /, \mid \times / \\
\mathrm{e} / \mathrm{r} /\end{array}$ & 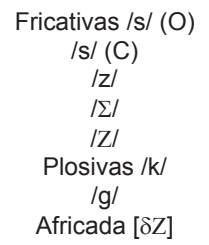 & $\begin{array}{c}57,14 \\
62,50 \\
38,57 \\
77,80 \\
25,00 \\
3,70 \\
0,00 \\
87,50\end{array}$ & $\begin{array}{c}97,44 \\
100,00 \\
96,00 \\
47,37 \\
37,50 \\
16,86 \\
4,76 \\
100,00\end{array}$ \\
\hline & O3 & $\begin{array}{c}\text { Líquidas /r/ e } \\
\qquad / / /\end{array}$ & $\begin{array}{c}\text { Plosiva /k/ } \\
\text { /g/ } \\
\text { Fricativa /s/ } \\
\text { /z/ }\end{array}$ & $\begin{array}{c}4,54 \\
12,50 \\
78,94 \\
72,72\end{array}$ & $\begin{array}{c}36,36 \\
85,71 \\
100,00 \\
100,00\end{array}$ \\
\hline
\end{tabular}

Legenda: Al: avaliação inicial. AF: avaliação final. O: onset. C: coda. *não houve possibilidade de ocorrência devido as demais classes não apresentarem alterações. 
Tabela 4 - Generalização para outra classe de sons no tratamento do grupo Leve-Moderado e Leve

\begin{tabular}{|c|c|c|c|c|c|}
\hline \multirow{2}{*}{ Modelo } & \multirow{2}{*}{ Sujeito } & \multirow{2}{*}{$\begin{array}{c}\text { Classes } \\
\text { estimuladas }\end{array}$} & \multirow{2}{*}{$\begin{array}{l}\text { Classes não } \\
\text { estimuladas }\end{array}$} & \multicolumn{2}{|c|}{$\%$ de acertos } \\
\hline & & & & $\mathrm{Al}$ & $\mathrm{AF}$ \\
\hline \multirow{6}{*}{ Leve - Moderado } & C5 & $\begin{array}{l}\text { Plosiva /g/ } \\
\text { Líquida /r/ }\end{array}$ & * & * & * \\
\hline & $\mathrm{C} 6$ & $\begin{array}{l}\text { Plosiva /g/ } \\
\text { Fricativa /Z/ } \\
\text { Líquida /r/ }\end{array}$ & Africada [ $\delta \mathrm{Z}]$ & 45,45 & 100,00 \\
\hline & A5 & Fricativa /Z/ & 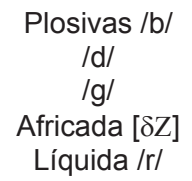 & $\begin{array}{l}63,16 \\
62,50 \\
46,15 \\
57,14 \\
92,00\end{array}$ & $\begin{array}{l}98,08 \\
98,25 \\
88,46 \\
92,86 \\
96,55\end{array}$ \\
\hline & A6 & Líquida /r/ & $\begin{array}{c}\text { Fricativas /f/ } \\
\text { /v/ } \\
\text { /s/ (O) } \\
\text { /s/ (C) } \\
\text { /z/ } \\
\mid \Sigma / \\
\text { /Z/ }\end{array}$ & $\begin{array}{c}28,57 \\
30,00 \\
61,11 \\
58,33 \\
76,92 \\
0,00 \\
0,00\end{array}$ & $\begin{array}{c}100,00 \\
92,00 \\
100,00 \\
87,50 \\
100,00 \\
100,00 \\
100,00\end{array}$ \\
\hline & 05 & $\begin{array}{l}\text { Fricativa } / z / \\
\text { Líquida } / \times 1\end{array}$ & * & * & * \\
\hline & 06 & $\begin{array}{l}\text { Plosiva /g/ } \\
\text { Fricativa /Z/ }\end{array}$ & 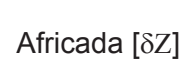 & 40,00 & 85,71 \\
\hline \multirow{6}{*}{ Leve } & C7 & Líquida /r/ & * & * & * \\
\hline & $\mathrm{C} 8$ & $\begin{array}{l}\text { Fricativa /f/ } \\
\text { Líquida /r/ }\end{array}$ & 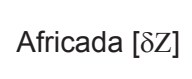 & 100,00 & 75,00 \\
\hline & A7 & $\begin{array}{l}\text { Fricativa /Z/ } \\
\text { Líquida /r/ }\end{array}$ & $\begin{array}{c}\text { Líquida /r/ } \\
\qquad / \times / \\
\text { Fricativas /Z/ } \\
\mid \Sigma / \\
/ \mathrm{s} /\end{array}$ & $\begin{array}{l}13,72 \\
46,15 \\
18,18 \\
30,56 \\
64,58\end{array}$ & $\begin{array}{l}86,93 \\
80,36 \\
92,31 \\
96,87 \\
93,33\end{array}$ \\
\hline & A8 & Líquida /r/ & $\begin{array}{c}\text { Plosiva /g/ } \\
\text { Fricativas /s/ } \\
/ \Sigma / \\
\text { |Zl }\end{array}$ & $\begin{array}{l}76,00 \\
77,78 \\
69,23 \\
45,46\end{array}$ & $\begin{array}{l}100,00 \\
100,00 \\
100,00 \\
100,00\end{array}$ \\
\hline & 07 & $\begin{array}{l}\text { Líquida /r/ } \\
\text { Nasal /n/ }\end{array}$ & * & * & * \\
\hline & 08 & $\begin{array}{l}\text { Africada }[\tau \Sigma] \\
\text { Líquida } / \times /\end{array}$ & $\begin{array}{c}\text { Fricativas /s/ } \\
\mid \mathrm{z} /\end{array}$ & $\begin{array}{l}50,00 \\
75,00\end{array}$ & $\begin{array}{r}87,50 \\
100,00\end{array}$ \\
\hline
\end{tabular}

Legenda: Al: avaliação inicial. AF: avaliação final. O: onset. C: coda. *não houve possibilidade de ocorrência devido as demais classes não apresentarem alterações.

moderado-grave, embora outros sujeitos apresentassem generalizações importantes para a evolução da terapia.

$\mathrm{Na}$ Tabela 4, encontram-se os resultados da generalização para outra classe de sons encontrada nos sujeitos com desvio leve-moderado e leve, submetidos aos modelos: Ciclos Modificado, ABAB-Retirada e Provas Múltiplas e Oposições Máximas Modificado.
Vê-se que a maioria dos sujeitos apresentou generalizações para as outras classes de sons. Os sujeitos C5, O5, C7 e $\mathrm{O} 7$ não tiveram a possibilidade de generalizar, pois as demais classes não apresentavam alterações. Enquanto que o C6, que foi estimulado com plosiva, líquida e fricativa, generalizou para a africada. A partir do tratamento com a fricativa, o A5 generalizou para as plosivas, africada e líquida. O A6 foi o que mais ampliou seu 
sistema fonológico, generalizou para as fricativas atingindo em alguns sons $100 \%$ de produções corretas. O outro sujeito $(\mathrm{O} 6)$ apresentou um aumento de produções corretas para a africada depois de ser estimulado com a plosiva e fricativa. O A8, tratado com a líquida $/ r /$, apresentou $100 \%$ de produções corretas para as fricativas /s/, /S/ e /3/ e para a plosiva /g/ na AFF. O sujeito O8, tratado pelo Modelo de Oposições Máximas Modificado, apresentou um aumento de produções corretas para as fricativas /s/ e /z/ depois de ser estimulado com a africada [t $\mathrm{f}]$ e a líquida / $K /$.

\section{DISCUSSÃO}

Em relação à generalização dentro de uma classe de sons, pode-se verificar que a maioria dos sujeitos apresentou um aumento das produções corretas de segmentos pertencentes à mesma classe dos sonsalvo, em todos os modelos de terapia pesquisados. Este tipo de generalização foi encontrado em três sujeitos pesquisados ${ }^{8} \mathrm{em}$ abordagens terapêuticas diferentes (Ciclos Modificado, ABAB-Retirada Provas Múltiplas e Oposições Máximas). Outro estudo ${ }^{3}$ encontrou a generalização dentro de uma classe de sons para todos os sujeitos estudados, enquanto que para outra classe de sons foi constatada em apenas dois dos sujeitos.

Os quatros sujeitos com desvio grave apresentaram importantes aumentos nas produções corretas para sons da mesma classe estimulada. Como se observa, alguns sujeitos (A2, O1 e O2) generalizaram para a líquida / $R /$, porém, um estudo ${ }^{13}$ referiu que não encontrou esse tipo de generalização em um dos sujeitos tratados com o rótico /R/, pelo Modelo Oposições Máximas Modificado. Quanto à líquida /r/, para o A1 e 01, assim como em outro estudo ${ }^{10}$ não foi encontrada generalização para a mesma classe de sons.

Dos sujeitos com desvio moderado-grave, os que demonstraram um maior aumento do percentual de produções corretas foram o $\mathrm{O} 3$, seguido do $\mathrm{A} 3$ e A4 tratados com Modelos Oposições Máximas Modificado e ABAB-Retirada e Provas Múltiplas. O estudo 20 com o Modelo de Oposições Máximas encontrou que a generalização dentro de uma classe de sons foi maior no grupo com desvio moderado-grave, seguido do grupo com desvio leve-moderado, do grupo com desvio grave e do grupo com desvio leve.

Nos sujeitos com desvio leve-moderado e leve verificou-se aumento das produções corretas dos sons pertencentes à mesma classe dos sons-alvo, em todos os modelos de terapia pesquisados. Este tipo de generalização também foi observado nos sujeitos estudados ${ }^{8}$ comparando os Modelos
Ciclos Modificado, ABAB-Retirada Provas Múltiplas e Oposições Máximas Modificado.

Quanto à líquida $/ r /$, o A6 concorda com um estudo ${ }^{10}$ que referiu não ter encontrado generalização para a mesma classe de sons (para a líquida não-lateral /r/).

Quanto à generalização para outras classes de sons, todos os sujeitos com desvio grave ou desvio moderado-grave apresentaram um aumento das produções corretas para a maioria dos sons pertencentes a outras classes de sons. O A1 generalizou para as classes não estimuladas das plosivas, nasais, fricativas e africadas. No entanto, a generalização para o A2 foi mais acentuada, pois houve um aumento de produções corretas para as classes das plosivas, nasais, fricativas e africadas.

O C4, a partir do tratamento com as líquidas e fricativas, generalizou apenas para a nasal e diminuiu o percentual de acertos para a classe das africadas. Um estudo ${ }^{1}$ não observou este tipo de generalização no sujeito submetido ao Modelo de Ciclos Modificado.

A partir do tratamento com a líquida e plosiva, - A3 obteve significativas generalizações para a classe não estimulada das fricativas, porém não generalizou para a africada. O A4 também ampliou seu sistema fonológico para as classes das plosivas, fricativas e africadas, por meio da estimulação com as líquidas. $\mathrm{O}$ sujeito $(\mathrm{O} 3)$ apresentou um aumento de produções corretas para as classes das plosivas e fricativas. Como pode ser observado nos sujeitos A1, A2, A3, A4 e O3 em relação à classe das fricativas, outro estudo ${ }^{10}$ também constatou a generalização para essa classe de sons (fricativas).

Nos grupos com desvio leve-moderado e leve, a maioria dos sujeitos apresentou generalizações para as outras classes de sons. No que se refere a este tipo de generalização nas diferentes gravidades do DF, uma autora ${ }^{21}$ verificou que esta generalização foi maior no grupo com desvio leve-moderado, seguido do grupo com desvio moderado-grave, do grupo com desvio grave e, por último, do grupo com desvio leve.

O A4 e o C8, ao contrário do C4, generalizaram para a classe da africada corroborando os achados de outro estudo ${ }^{1}$. Enquanto que para os sujeitos C5, O5, C7 e O7 não foram observados generalização para outras classes de sons, pois não apresentavam dificuldades em outras classes de sons além das estimuladas, esse fato também foi relatado em outra pesquisa ${ }^{3}$.

Dessa forma, observou-se que os sujeitos submetidos ao Modelo ABAB-Retirada e Provas Múltiplas foram os que apresentaram um maior número de produções correta, sendo no grupo com desvio grave, o A2; no grupo com desvio moderado-grave, 
o A3; no grupo com desvio leve-moderado, o A6; e no grupo com desvio leve, o A7. Ao contrário de uma pesquisa ${ }^{8} \mathrm{com}$ três sujeitos submetidos aos Modelos Oposições Máximas, ABAB-Retirada e Provas Múltiplas e Ciclos Modificado verificou que apenas o sujeito tratado com o Modelo Oposições Máximas generalizou para a outra classe de sons (plosivas).

\section{CONCLUSÃO}

Com base nos resultados desta pesquisa verificou-se que a aplicação dos modelos terapêuticos foi eficaz no tratamento dos sujeitos com DF, pois a maioria dos sujeitos apresentou generalização dentro de uma classe de sons e para outras classes de sons.

A generalização dentro de uma classe de sons ocorreu em todos os modelos pesquisados, porém, foi observada principalmente nos sujeitos submetidos aos Modelos de ABAB-Retirada e Provas Múltiplas e Oposições Máximas Modificado.

A generalização para outras classes de sons foi observada em todos os modelos estudados, porém, os sujeitos submetidos ao Modelo ABAB-Retirada e Provas Múltiplas foram os que apresentaram um maior número de produções corretas para as diferentes gravidades do DF.

\begin{abstract}
Purpose: to compare the generalization inside of a sound class and for other sound classes in the treatment of subjects with different severity levels of phonological disorders submitted to the Modified Cycles Model, ABAB-Withdrawal and Multiple Probes and Maximum Opposition Model. Methods: 21 children with phonological disorder, 11 males and 10 females, with average age 5:7, were their speech data analyzed through the phonological assessment. From the contrastive analysis we calculated the Percentage of Correct Consonants (Shriberg \& Kwiatkowski, 1982) and the subjects were classified in the different severity levels of phonological disorders: severe, moderate-severe, mild-moderate and mild. Six subjects were submitted to the treatment with Modified Cycles Model, eight to the ABABWithdrawal and Multiple Probes Model, and seven to the Modified Maximum Opposition Model. After this was compared the generalization inside of a sound class and for other sound classes among three therapy models for each severity level of phonological disorder. Results: the generalization inside a sound class occurred in all studied models. ABAB-Withdrawal and Multiple Probes Model were effective for all severity groups and the Modified Maximum Opposition were more effective for the severe and moderate-severe groups. In the generalization for other sound classes, the subjects treated with ABAB-Withdrawal and Multiple Probes Model showed a major generalization in different severity levels of phonological disorder. Conclusion: all the subjects showed evolution in all kinds of generalization studied in three different therapeutic models.
\end{abstract}

KEYWORDS: Speech Therapy; Generalization; Speech; Speech Disorders

\section{REFERÊNCIAS}

1. Mota HB, Pereira LF. A generalização na terapia dos desvios fonológicos: experiência com duas crianças. Pró-Fono. 2001; 13(2):141-6.

2. Mota HB, Bagetti T, Keske-Soares M, Pereira LF. A generalização baseada nas relações implicacionais em sujeitos submetidos à terapia fonológica. PróFono. 2005; 17(1):99-110.

3. Mota HB, Bagetti T, Keske-Soares M, Pereira LF. A generalização em sujeitos com desvio fonológico médio-moderado tratados pelo modelo de oposições máximas. Rev Soc Bras Fonoaudiol. 2004; 9(2):102-11.
4. Elbert M, Gierut JA. Handbook of clinical phonology. London: Taylor \& Francis; 1986.

5. Tyler AA, Edwards ML, Saxman JH. Clinical application of two phonological treatment procedures. J Speech Hear Disord. 1987; 52(4):393-409.

6. Tyler A, Figursky GR. Phonetic inventory chances after treating distinctions along implicatonal hierarchy. Clin Linguist Phon. 1994; 8:91-107.

7. Bagetti T, Mota HB, Keske-Soares M. Modelo de oposições máximas modificado: uma proposta de tratamento para o desvio fonológico. Rev Soc Bras Fonoaudiol. 2005; 10(1):36-47.

8. Mota HB, Keske-Soares M, Ferla A, Zasso LV, Dutra LV. Estudo comparativo da generalização em 
três modelos de terapia para desvios fonológicos. Saúde. 2002; 26(1/2):36-47.

9. Hodson BW. Identifying phonological patterns and projecting remediation cycles: expediting intelligibility gains of a 7 year old Australian child. Adv Speech-Lang Pathol. 2006; 8(3):257-64.

10. Barberena L, Keske-Soares M, Mota HB. Generalização no tratamento com o/R/ em um caso de desvio fonológico médio-moderado. Rev Soc Bras Fonoaudiol. 2004; 9(4):229-36.

11. Williams AL. A systematic perspective for assessment and intervention: a case study. Adv Speech-Lang Pathol. 2006; 8(3):245-56.

12. Hayden D. The PROMPT model: use and application for children with mixed phonologicalmotor impairment. Adv Speech-Lang Path. 2006; 8(3):265-81.

13. Crosbie S, Pine C, Holm A, Dodd B. Treating jarrod: a core vocabulary approach. Adv SpeechLang Pathol. 2006; 8(3): 316-21.

14. McLeod S. An holistic view of a child with unintelligible speech: insights from the ICF and ICFCY. Adv Speech-Lang Pathol. 2006; 8(3):293-315.

15. Crosbie S, Holm A, Dodd B. Intervention for children with severe speech disorder: a comparison of two approaches. Int J Lang Commun Dis. 2005; 40(4):467-91.

16. Mota HB, Keske-Soares M, Bagetti T, Ceron MI, Melo Filha MGC. Análise comparativa da eficiência de três diferentes modelos de terapia fonológica. Pró-Fono. 2007; 19(1):67-74.
17. Ceron MI, Keske-Soares M. Terapia fonológica: a generalização a itens não utilizados no tratamento (outras palavras). Rev CEFAC. 2007; 9(4):453-60.

18. Blanco AP. A generalização no modelo de ciclos modificado em pacientes com diferentes graus de severidade de desvio fonológico [mestrado]. Santa Maria (RS): Universidade Federal de Santa Maria; 2003.

19. Barberena L. A generalização obtida pelo Modelo "ABAB - Retirada e Provas Múltiplas" em diferentes graus de severidade do desvio fonológico [mestrado]. Santa Maria (RS): Universidade Federal de Santa Maria; 2005.

20. Donicht G. A generalização obtida a partir do tratamento como os róticos em dois modelos de terapia fonoaudiológica para crianças com desvios fonológicos [monografia]. Santa Maria (RS): Universidade Federal de Santa Maria (UFSM); 2005.

21. Bagetti T. Mudanças fonológicas em sujeitos com diferentes graus de severidade do desvio fonológico tratados pelo modelo de oposições máximas modificado [mestrado]. Santa Maria (RS): Universidade Federal de Santa Maria; 2005.

22. Shriberg LD, Kwiatkowski J. Phonological disorders I: a diagnostic classification system. J Speech Hear Disord. 1982; 47(3):226-41.

23. Yavas M, Hernandorena CLM, Lamprecht RR. Avaliação fonológica da criança: reeducação e terapia. Porto Alegre: Artes Médicas; 1991.148p.

24. Bernhardt B. Developmental implications of nonlinear phonological theory. Clin Linguist Phon. 1992; 6:259-81.

RECEBIDO EM: 15/8/2007

ACEITO EM: 25/04/2008

Endereço para correspondência:

Rua Bentevi, 215

Santa Maria - RS

CEP: $97035-130$

Tel: (55) 9985-6067

E-mail: marizeteceron@ hotmail.com 